04.1

\title{
Особенности формирования собственного электрического поля низкотемпературной кислород-метановой плазмы
}

\author{
() А.В. Рудинский, Д.А. Ягодников, С.В. Рыжков, В.В. Онуфриев \\ Московский государственный технический университет им. Н.Э. Баумана, Москва, Россия \\ E-mail: ravman@bmstu.ru
}

Поступило в Редакцию 27 ноября 2020г.

В окончательной редакции 9 февраля 2021 г.

Принято к публикации 25 фревраля 2021 г.

\begin{abstract}
Разработана математическая модель горения кислород-метановой плазмы при давлениях $0.2-1.6 \mathrm{MPa} \mathrm{c}$ учетом ионизации продуктов сгорания и рекомбинации заряженных компонентов. Определены концентрации положительных ионов для характерных зон реагирующей кислород-метановой смеси в предположении амбиполярной диффузии. Получены распределения напряженности самосогласованного электрического поля и генерируемого электрического заряда по длине канала. Результаты моделирования по уровню концентраций электронов и ионов в пламени верифицированы известными экспериментальными данными, полученными зондовыми методами в условиях работы модельного жидкостного ракетного двигателя.
\end{abstract}

Ключевые слова: низкотемпературная плазма, кислород, метан, горение, амбиполярная диффузия, собственное электрическое поле.

DOI: 10.21883/PJTF.2021.10.50973.18638

Преобразование химической энергии в тепловую в камере жидкостных ракетных двигателей (ЖРД) происходит в процессе горения топлива и характеризуется первичной генерацией заряженных компонентов (ионов и электронов) и экзотермическими реакциями рекомбинации, протекающими преимущественно за фронтом пламени. Продукты сгорания топлива находятся в камере сгорания (КС) при высоких давлениях (более $25 \mathrm{MPa}$ ) и температурах (более $3500 \mathrm{~K}$ ), характерных для ЖРД, и могут рассматриваться как квазинейтральная плазма. При этом для плазмы продуктов сгорания углеводородного топлива характерны зоны с распределением заряженных компонентов (ионов и электронов) с преобладающим механизмом амбиполярной диффузии над тепловым [1]. Такое разделение зарядов разных знаков обеспечивается самосогласованным электрическим полем, напряженность которого достигает значений $1350 \mathrm{~V} / \mathrm{m}$ и может обеспечивать скорость образования заряженных частиц одного знака $\sim 1.1 \cdot 10^{-4} \mathrm{C} /\left(\mathrm{cm}^{3} \cdot \mathrm{s}\right)$ во фронте горения метановоздушной смеси согласно [2]. Существенные отличия значений теоретических концентраций заряженных компонентов, полученных по данным термодинамического равновесного расчета, от результатов экспериментов, проводимых с помощью электростатических зондов [3,4], обусловливают необходимость учета при моделировании электрофизических процессов диффузионных потоков ионов и электронов в плазме продуктов сгорания для характерных областей камеры сгорания ЖРД, создающих собственное электрическое поле, напряженность которого может быть зарегистрирована специальными датчиками [5].

Моделирование собственного электрического поля в прифронтовой области горения в КС необходимо для определения механизмов электризации твердых частиц сажи $[6,7]$ или металлов $[8,9]$ (последние могут образовываться в потоке в результате начала разрушения стенок проточного тракта ЖРД из-за тепловой эрозии), что положено в основу создания бесконтактных методов технической диагностики. Цель настоящей работы заключается в расчетно-теоретическом исследовании разделения носителей электрических зарядов и определении характеристик собственного электрического поля углеводородных продуктов сгорания применительно к условиям, реализуемым в камере жидкостного ракетного двигателя.

Математическое моделирование включало в себя три основные стадии.

1. В результате осесимметричного расчета в программе Ansys Fluent определялись химический состав реагирующей смеси кислород-метан и распределение температуры по цилиндрической КС.

2. По найденным распределениям концентраций электронейтральных компонентов смеси $\mathrm{H}_{2} \mathrm{O}, \mathrm{CH}, \mathrm{O}, \mathrm{CO}_{2}$ определялись концентрации заряженных компонентов (положительных ионов $\mathrm{H}_{3} \mathrm{O}^{+}$и электронов) в соответствии с реакциями ионизации

$$
\begin{gathered}
\mathrm{CH}+\mathrm{O} \rightarrow \mathrm{CHO}^{+}+e, \\
\mathrm{CHO}^{+}+\mathrm{H}_{2} \mathrm{O} \rightarrow \mathrm{H}_{3} \mathrm{O}^{+}+\mathrm{CO}, \\
{\left[\mathrm{CHO}^{+}\right]=\frac{k_{1}[\mathrm{CH}] \cdot[\mathrm{O}]}{k_{2}\left[\mathrm{H}_{2} \mathrm{O}\right]},}
\end{gathered}
$$

где $\left[\mathrm{CHO}^{+}\right],[\mathrm{CH}],[\mathrm{O}],\left[\mathrm{H}_{2} \mathrm{O}\right]-$ мольные концентрации соответствующих компонентов продуктов сгорания, $k_{1}$ - константа химической реакции (1), $k_{2}$ - константа химической реакции (2). 
В качестве заряженных компонентов смеси продуктов сгорания кислород-метан были выбраны положительные ионы $\mathrm{H}_{3} \mathrm{O}^{+}$и электронный газ, так как данные компоненты обладают наибольшими концентрациями по результатам предварительных равновесных термодинамических расчетов.

3. Определялась напряженность электрического поля по длине камеры сгорания в соответствии с найденными распределениями химического состава, температуры и характерными зонами: I - зона предварительного подогрева исходной смеси кислород-метан; II - зона ионизации продуктов сгорания в области максимальной температуры и ее окрестности; III - зона рекомбинации электронов и ионов в продуктах сгорания в соответствии с химической реакцией

$$
\mathrm{H}_{3} \mathrm{O}^{+}+e \rightarrow \mathrm{H}_{2} \mathrm{O}+\mathrm{H}
$$

Система уравнений, определяющая концентрации заряженных компонентов (ионов и электронов) в плазме продуктов сгорания, состояла из уравнений сохранения диффузионных потоков положительных ионов и электронов. Напряженность самосогласованного электрического поля описывалась уравнением Пуассона

$$
\begin{gathered}
\frac{d}{d x}\left(u n_{i}-D_{i} \frac{d n_{i}}{d x}+b_{i} n_{i} E\right)=\frac{d j_{i}}{d x}=W_{t}-k_{r} n_{i} n_{e} \\
\frac{d}{d x}\left(u n_{e}-D_{e} \frac{d n_{e}}{d x}-b_{e} n_{e} E\right)=\frac{d j_{e}}{d x}=W_{t}-k_{r} n_{i} n_{e} \\
\frac{d E}{d x}=\frac{e}{\varepsilon_{0}}\left(n_{i}-n_{e}\right)
\end{gathered}
$$

где $u$ - скорость $[\mathrm{m} / \mathrm{s}], n_{i}-$ объемная концентрация положительных ионов $\left[\mathrm{m}^{-3}\right], b_{i}$ - подвижность ионов $\left[\mathrm{m}^{2} /(\mathrm{V} \cdot \mathrm{s})\right], E-$ напряженность самосогласованного электрического поля $[\mathrm{V} / \mathrm{s}], j_{i}$ - диффузионный поток ионов $\left[\left(\mathrm{m}^{2} \cdot \mathrm{s}\right)^{-1}\right], W_{t}$ - скорость образования ионов, $k_{r}$ - константа реакции рекомбинации $\left[\mathrm{m}^{3} / \mathrm{s}\right], n_{e}-$ объемная концентрация электронов $\left[\mathrm{m}^{-3}\right], b_{e}-$ подвижность электронов $\left[\mathrm{m}^{2} /(\mathrm{V} \cdot \mathrm{s})\right], j_{e}-$ диффузионный поток электронов $\left[\left(\mathrm{m}^{2} \cdot \mathrm{s}\right)^{-1}\right], e-$ заряд электрона $[\mathrm{C}], \varepsilon_{0}$ - электрическая постоянная $\left[\mathrm{m}^{-3} \cdot \mathrm{kg}^{-1} \cdot \mathrm{s}^{2} \cdot \mathrm{C}^{2}\right]$, $x$ - осевая координата $[\mathrm{m}], D_{e}, D_{i}$ - коэффициенты диффузии электронов и ионов $\left[\mathrm{m}^{2} / \mathrm{s}\right]$.

Распределение напряженности самосогласованного поля по осевой координате КС определялось интегрированием системы (5)-(7) с граничными условиями в соответствии с характерными зонами горения плазмы с учетом химических реакций (1)-(4).

Механизм образования собственного электрического поля в рассматриваемой модели заключается в разделении зарядов вследствие разной подвижности и коэффициентов диффузии положительных ионов $D_{i}$ и электронов $D_{e}$ (режим амбиполярной диффузии). В этом случае для плазмы в зоне горения величина радиуса Дебая должна быть меньше характерного размера прогретого слоя, т.е.

$$
R_{\mathrm{D}} \ll \mathrm{D} / \mathrm{u},
$$

где $D-$ коэффициент диффузии $\left[\mathrm{m}^{2} / \mathrm{s}\right], R_{\mathrm{D}}-$ радиус Дебая $[\mathrm{m}]$, определяемый исходя из зависимости

$$
R_{\mathrm{D}}=\sqrt{\frac{\varepsilon_{0} k T}{e^{2} n_{e}}} .
$$

В результате предварительных оценок для кислород-метановой плазмы радиус Дебая составил $R_{\mathrm{D}}=(20.2-5.4) \cdot 10^{-6} \mathrm{~m}$, что на несколько порядков меньше ширины фронта горения указанных компонентов топлива. Оценки радиуса Дебая проводились в предположении равновесных концентраций электронов для температур $T_{0}=3098-3366 \mathrm{~K} \quad$ и давлений $p_{0}=0.2-1.6 \mathrm{MPa}$, характерных для режимов горения в камере сгорания ЖРД малой тяги [4]. Равновесные объемные концентрации электронов определялись в программе Terra [10] в зависимости от коэффициента избытка окислителя (кислорода) и давления в КС (см. таблицу). Проведенные оценки показали, что распределения положительных ионов и электронов в окрестности фронта горения могут быть найдены в предположении амбиполярной диффузии (с учетом равенства диффузионных потоков).

С целью определения особенностей структуры течения (распределение температуры и скорости) с учетом смешения метана с кислородом проведено предварительное численное моделирование. Моделирование газовой динамики и тепломассообмена при течении и взаимодействии двух заранее не перемешанных газовых потоков основано на решении системы уравнений сохранения массы, импульса и энергии, а также уравнений переноса компонентов потока в осесимметричной постановке. Система уравнений после осреднения по Фавру произвольных параметров потока (температура, скорость и т.д.) дополнялась уравнениями состояния идеального газа и модели турбулентности $k-\varepsilon$. Процессы горения моделировались с помощью редуцированного кинетического механизма, основанного на детальном механизме GRI-MECH 3.0 [11].

Объектом моделирования являлась цилиндрическая КС модельного кислород-метанового ЖРД малой тяги [4] диаметром $35 \mathrm{~mm}$ и длиной $120 \mathrm{~mm}$. Распределения теплофизических параметров на рис. 1 получены при стехиометрическом соотношении и давлении

Параметры плазмы жидкий кислород + жидкий метан при стехиометрическом соотношении

\begin{tabular}{c|c|c|c}
\hline$p_{0}, \mathrm{MPa}$ & $n_{e}, 10^{18} \mathrm{~m}^{-3}$ & $T_{0}, \mathrm{~K}$ & $R_{\mathrm{D}}, 10^{-6} \mathrm{~m}$ \\
\hline 0.2 & 0.0363 & 3098 & 20.16 \\
0.4 & 0.0925 & 3185 & 12.81 \\
0.8 & 0.2290 & 3274 & 8.25 \\
1.6 & 0.5489 & 3366 & 5.40
\end{tabular}




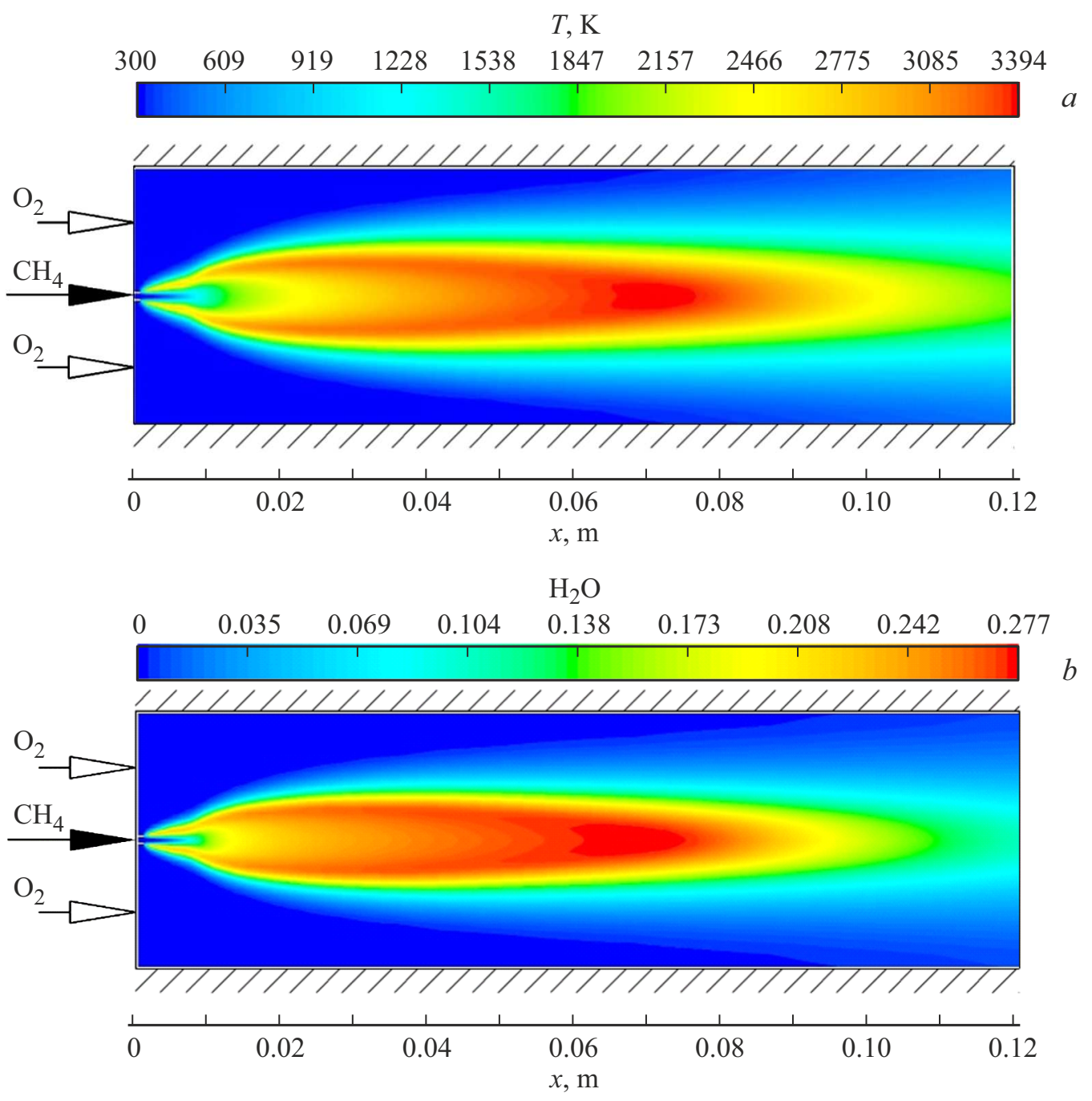

Рис. 1. Распределение теплофизических параметров по КС. $a-$ температура, $b-$ массовая доля $\mathrm{H}_{2} \mathrm{O}$.

$0.8 \mathrm{MPa.} \mathrm{Конечно-элементная} \mathrm{модель} \mathrm{КС} \mathrm{[4]} \mathrm{представ-}$ ляла собой структурированную сетку с $~ 10000$ ячеек. Подача метана осуществлялась через центральную форсунку диаметром $4 \mathrm{~mm}$, кислорода - через две внешние кольцевые щели (рис.1, $a$ ).

Из рис. 2 видно, что напряженность собственного электрического поля (НСЭП) пламени убывает и достигает минимального значения. Убывание НСЭП до отрицательных значений происходит в зоне предварительного подогрева топливной смеси, характеризующейся шириной $x_{\mathrm{I}}=D_{i} / u \quad\left(x_{\mathrm{I}} \approx 0.1 \mathrm{~mm}\right.$ при $\left.u=10 \mathrm{~m} / \mathrm{s}\right)$. Концентрации электронов и ионов в области предварительного подогрева равны, и перенос заряженных компонентов осуществляется за счет механизма амбиполярной диффузии, т.е. $R_{\mathrm{D}} u / D_{i} \ll 1$. Достигаемое минимальное значение напряженности электрического поля $E_{\min }=-u /\left(2 b_{i}\right) \approx-1000 \mathrm{~V} / \mathrm{m}$ обеспечивает равные диффузионные потоки и, следовательно, концентрации электронов и положительных ионов (рис. 3), при том что подвижности и коэффициенты диффузии соотносятся как
$D_{i} / D_{e}=b_{i} / b_{e} \approx 10^{-3}$ при $D_{i}=(1.2-1.6) \cdot 10^{-3} \mathrm{~m}^{2} / \mathrm{s}$. По длине фронта горения НСЭП увеличивается и становится положительной, достигая максимального значения (рис. 2). Это объясняется уменьшением влияния собственного самосогласованного электрического поля на

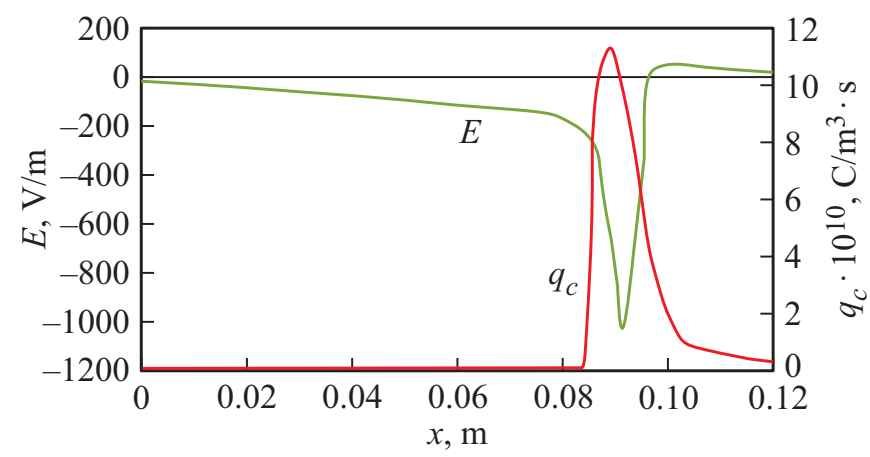

Рис. 2. Распределение по длине КС в осевом сечении напряженности собственного электрического поля $E$ и скорости образования заряженных компонентов одного знака $q_{c}$. 


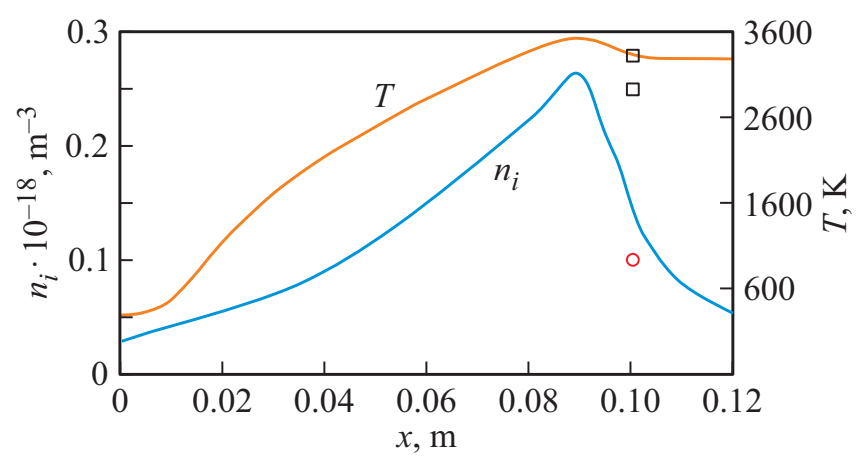

Рис. 3. Распределение положительных ионов $\mathrm{H}_{3} \mathrm{O}^{+}$и температуры по длине КС в сечении по оси симметрии. Кружок равновесное значение, квадраты - экспериментальные результаты [4].

механизм диффузии, что сопровождается увеличением радиуса Дебая вследствие роста температуры из-за протекания химических реакций горения, и переход в режим свободной диффузии электронов и ионов. Максимальное значение НСЭП в этой области соответствует положительной величине $E_{\max }=D_{i} k_{T} n_{e \infty} / u \approx 50 \mathrm{~V} / \mathrm{m}$ при константе скорости $k_{T} \approx 10^{-6} \mathrm{~m}^{3} / \mathrm{s}$. В области, характеризующейся продуктами сгорания (за фронтом горения), НСЭП уменьшается, стремясь к нулю, что объясняется уменьшением концентрации заряженных компонентов из-за реакций рекомбинации ионов и электронов. Радиус Дебая в рекомбинационной области увеличивается пропорционально $n_{e}^{-0.5}$, и влияние амбиполярной диффузии на формирование собственного электрического поля пропадает по мере удаления от фронта горения.

Проведенные таким образом фундаментальные исследования позволили выявить области кислородметанового пламени, в которых реализуются максимальные значения концентраций положительно заряженных ионов, суммарного электрического заряда и напряженности электрического поля, которые обусловливаются пространственным распределением температуры и концентраций положительных и отрицательных заряженных ионов.

\section{Финансирование работы}

Работа выполнена при поддержке Госпрограммы № 0705-2020-0044 фундаментальных исследований лаборатории „Внутрикамерные процессы ракетных и реактивных двигателей“.

\section{Конфликт интересов}

Авторы заявляют, что у них нет конфликта интересов.

\section{Список литературы}

[1] Н.И. Кидин, Г.М. Махвиладзе, Физика горения и взрыва, № 6, 865 (1976).

[2] К.Е. Улыбышев, Изв. РАН, Механика жидкости и газа, № 1,3 (1998).

[3] G. Maise, A.J. Sabadell, in AIAA 5th Propulsion Joint Specialist Conf. (Colorado Springs, CO, USA, 1969). https://doi.org/10.2514/6.1969-573

[4] J. Nichol, V. Siminski, H.G. Wolfhard, Symposium (International) on Combustion, 8 (1), 235 (1961).

[5] С.А. Гришин, Г.А. Пашкевич, В.А. Селянтьев, Н.С. Недвецкий, А.А. Бунчук, К.Ю. Желудевич, В.В. Климентовский, Д.А. Ягодников, В сб. науч. тр. V Конгресс физиков Беларуси (Ковчег, Минск, 2015), с. 241.

[6] A. Sorokin, X. Vancassel, P. Mirabel, Atmos. Chem. Phys., 3, 325 (2003).

[7] А.М. Савельев, А.М. Старик, ЖТФ, 76 (4), 53 (2006).

[8] Я.А. Лялин, К.И. Семенов, Н.Х. Копыт, в сб. Физика аэродисперсных систем (Одесса, 2012), № 49, с. 112.

[9] А.В. Рудинский, Д.А. Ягодников, ТВТ, 57 (5), 777 (2019). DOI: $10.1134 / \mathrm{S} 0040364419050132$

[10] B.G. Trusov, A.G. Malanichev, in 3rd Int. Conf. on chemical kinetics (Washington, 1993), p. 56-57.

[11] G.P. Smith, D.M. Golden, M. Frenklach, N.W. Moriarty, B. Eiteneer, M. Goldenberg, C.T. Bowman, R.K. Hanson, S. Song, W.C. Gardiner, Jr., V.V. Lissianski, Z. Qin, GRI-MECH 3.0 [Электронный ресурc]. Режим доступа: http://combustion.berkeley.edu/gri-mech/version30/ text30.html 